\title{
Dietary intakes of calcium, iron and vitamin $D$ in club and county female Gaelic footballers
}

\author{
E. Gibbons and G. Mc Monagle \\ Department of Science, School of Science, Letterkenny Institute of Technology, Letterkenny, Co. Donegal, Ireland
}

Ladies Gaelic Football is recognised as one of the fastest growing female sports in Europe ${ }^{(1)}$. At present, there is a paucity of research exploring the dietary intakes of female Gaelic footballers, and an absence of sport specific nutrition guidelines. Inadequate intakes of calcium and iron are common in females ${ }^{(3)}$, with inadequate iron intakes previously documented in female team sports ${ }^{(4)}$. Vitamin D insufficiency is also evident in male Gaelic footballers but has yet to be examined in females ${ }^{(5)}$. Therefore, the aim of this study was to evaluate calcium, iron and vitamin D intakes of club and county female Gaelic footballers, and compare them to recommended dietary allowances (RDAs) ${ }^{(2)}$.

Dietary intakes of club and county level female Gaelic footballers were recorded using three-day food diaries, which also included intakes of dietary supplements. Club $(n=23)$ and county $(n=26)$ footballers were recruited from three female Gaelic football teams. Of those recruited initially, food diaries were completed and returned by $78 \%(n=18)$ of club and $74 \%(n=19)$ of county footballers, under-reporting was evident in $22 \%$ of participants who were subsequently excluded from analysis. The remaining food diaries $(n=29)$ were assessed using dietary analysis software to compare micronutrient intakes to RDAs ${ }^{(2)}$ and further analysed using SPSS version 24.

\begin{tabular}{|c|c|c|c|c|c|c|c|c|}
\hline \multirow{2}{*}{ Nutrients } & \multirow{2}{*}{ RDA } & \multicolumn{3}{|c|}{ County footballers $(n=16)$} & \multicolumn{3}{|c|}{ Club footballers $(n=13)$} & \multirow[b]{2}{*}{$P^{\mathrm{a}}$} \\
\hline & & Median & 25 th & 75 th & Median & 25 th & 75 th & \\
\hline Calcium (mg/d) & 800 & 692 & $581 \cdot 5$ & $736 \cdot 25$ & 480 & $413 \cdot 5$ & 665 & 0.42 \\
\hline $\operatorname{Iron}(\mathrm{mg} / \mathrm{d})$ & 14 & $12^{\mathrm{b}}$ & 10.73 & $13 \cdot 83$ & $9 \cdot 8^{\mathrm{b}}$ & $7 \cdot 09$ & $11 \cdot 55$ & 0.003 \\
\hline Vitamin $D(\mu \mathrm{g} / \mathrm{d})$ & 10 & $2 \cdot 7$ & 1.93 & 5.48 & $2 \cdot 9$ & $2 \cdot 2$ & $3 \cdot 95$ & $0 \cdot 277$ \\
\hline
\end{tabular}

${ }^{\mathrm{a}} P$ for difference between groups (independent t-test, $\left.P<0.05\right)$, ${ }^{\mathrm{b}}$ denotes significant differences between groups.

Overall, median intakes of calcium, iron and vitamin D in both club and county footballers were lower than the respective RDAs. A minority of county players $(n=2)$ met the RDAs for calcium, iron and vitamin D with the aid of dietary supplements. Furthermore, iron intakes of club players were significantly lower than county players $(P<0.05)$. However, there were no significant differences between club and county players in relation to calcium and vitamin $\mathrm{D}$ intakes $(P=0.42$ and $P=0 \cdot 277$, respectively).

In conclusion, this study highlights the prevalence of low calcium, iron and vitamin D intakes in club and county female Gaelic footballers, with intakes of iron particularly low in club players. These findings should be confirmed through serological investigation of such micronutrients in female Gaelic footballers. This study illustrates the need to raise awareness of the importance of sufficient micronutrient intakes in female Gaelic football players in order to reduce the risk of such deficiencies amongst this group of athletes.

1. Ladies Gaelic Football Association (no date) History: the beginning. http://ladiesgaelic.ie/about-lgfa/history/ (accessed March 2018).

2. Food Safety Authority of Ireland (1999) Recommended Dietary Allowances for Ireland. Dublin: FSAI.

3. Irish University Nutrition Alliance (IUNA) (2011) National Adult Nutrition Survey. Dublin: IUNA.

4. Ahmadi A, Enayatizadeh N, Akbarzadeh M et al. (2010) Pak J Biol Sci 13, 93-96.

5. Magee PJ, Pourshahidi LK, Leech L et al. (2012) Proc Nutr Soc 71(OCE2), E100. 